\title{
Evolutionary history and genetic connectivity across highly fragmented populations of an endangered daisy
}

\author{
Yael S. Rodger $\mathbb{( D}^{1} \cdot$ Alexandra Pavlova $\mathbb{( D}^{1} \cdot$ Steve Sinclair $^{2} \cdot$ Melinda Pickup $^{3} \cdot$ Paul Sunnucks $\mathbb{D}^{1}$
}

Received: 29 May 2020 / Revised: 24 January 2021 / Accepted: 25 January 2021 / Published online: 19 February 2021

(c) The Author(s) 2021. This article is published with open access

\begin{abstract}
Conservation management can be aided by knowledge of genetic diversity and evolutionary history, so that ecological and evolutionary processes can be preserved. The Button Wrinklewort daisy (Rutidosis leptorrhynchoides) was a common component of grassy ecosystems in south-eastern Australia. It is now endangered due to extensive habitat loss and the impacts of livestock grazing, and is currently restricted to a few small populations in two regions $>500 \mathrm{~km}$ apart, one in Victoria, the other in the Australian Capital Territory and nearby New South Wales (ACT/NSW). Using a genome-wide SNP dataset, we assessed patterns of genetic structure and genetic differentiation of 12 natural diploid populations. We estimated intrapopulation genetic diversity to scope sources for genetic management. Bayesian clustering and principal coordinate analyses showed strong population genetic differentiation between the two regions, and substantial substructure within ACT/ NSW. A coalescent tree-building approach implemented in SNAPP indicated evolutionary divergence between the two distant regions. Among the populations screened, the last two known remaining Victorian populations had the highest genetic diversity, despite having among the lowest recent census sizes. A maximum likelihood population tree method implemented in TreeMix suggested little or no recent gene flow except potentially between very close neighbours. Populations that were more genetically distinctive had lower genetic diversity, suggesting that drift in isolation is likely driving population differentiation though loss of diversity, hence re-establishing gene flow among them is desirable. These results provide background knowledge for evidence-based conservation and support genetic rescue within and between regions to elevate genetic diversity and alleviate inbreeding.
\end{abstract}

\section{Introduction}

Biodiversity is declining globally at an unprecedented rate with tens of thousands of species facing impending extinction (Pimm et al., 2015; IUCN, 2020). Genetic diversity is an essential part of biodiversity, supporting populations' persistence by promoting fitness and ability to

Supplementary information The online version contains supplementary material available at https://doi.org/10.1038/s41437021-00413-0.

Yael S. Rodger

yael.rodger@monash.edu

1 School of Biological Sciences, Clayton Campus, Monash University, Clayton, VIC, Australia

2 Arthur Rylah Institute, Department of Environment, Land Water and Planning, Heidelberg, VIC, Australia

3 Greening Australia Ltd, Perth, WA, Australia adapt evolutionarily (Lande \& Shannon, 1996). Thus, maintaining genetic diversity and evolutionary processes, such as gene flow and adaptive change, is critical for biodiversity conservation (Moritz, 1999; Crandall et al., 2000). Decreased gene flow under habitat fragmentation reduces the spread of novel genetic variants, lowering adaptive potential (Frankham et al., 2017). Genetic drift in small populations results in loss of genetic diversity and reduced efficiency of natural selection (Ellstrand \& Elam, 1993; Frankham et al., 1999), leading to inbreeding depression and lowered adaptive potential, which elevate extinction risk (Frankham, 2005; Frankham et al., 2014). Thus, conservation management must consider conserving and augmenting genetic diversity and gene flow (Weeks et al., 2011; Frankham et al., 2017; Ralls et al., 2018).

Understanding the evolutionary processes underpinning a species' distribution and population structure is essential for developing appropriate species-wide genetic management (Weeks et al., 2011; Pavlova et al., 2014). Both longterm divergence and short-term drift in recently isolated 
populations may result in population genetic structure. If populations are managed separately based on the incorrect assumption of genetic uniqueness rather than recent differentiation by genetic drift, extinction risk may increase by perpetuating the loss of genetic diversity through isolation and further drift (Coleman et al., 2013). Conversely, mixing populations that are too divergent can result in outbreeding depression, although this is infrequently observed, and its occurrence can usually be avoided by attention to risk factors including fixed chromosomal differences, adaptation to different environments and length of time since last gene flow (Frankham et al., 2011; Frankham 2015). Properly conducted genetic rescue is becoming widely accepted as a biodiversity conservation approach, particularly when the relative risks and benefits of mixing versus not mixing gene pools are properly assessed (Whiteley et al., 2015; Frankham et al., 2017; Ralls et al., 2018; Liddell et al., 2020). Accordingly, identifying the major processes underlying differentiation is important for informing risk-assessment frameworks and decision-support tools for maximising population persistence (Weeks et al., 2016). While these tools are increasingly available, and the data required more tractable to obtain, there remains much unfulfilled potential for evidence-based conservation decision making that embraces the importance of maintaining evolutionary processes (Liddell et al., 2020).

Understanding how best to reconnect fragmented populations requires an understanding of the levels of historical connectivity, which can be assessed using population genetic data (Mijangos et al., 2015; Breed et al., 2019). Research on genetic structure and past and present gene flow has been used to encourage admixture between populations of conservation concern, define seed transfer zones for restoring native grasslands and identify sources of genetic material to maximise evolutionary potential and increase restoration success (Knapp \& Rice, 1996; Diekmann et al., 2010; Lloyd et al., 2011; Pacioni et al., 2015; Rodger et al., 2018; Potter et al., 2020). Despite the potential for population genetics to guide effective management, understanding of past and present connectivity is missing for many species. Even when relevant data exist, it remains rare for management-relevant interpretations of population genetic data to be articulated in terms likely to be useful to managers (Liddell et al., 2020).

The Button Wrinklewort (Rutidosis leptorrhynchoides, also spelled leptorhynchoides) is a perennial plant in the daisy (Asteraceae) family, endemic to Australia, and that was once widespread in grassy ecosystems in south-eastern Australia (Scarlett \& Parsons, 1990). Since European human colonisation of its habitat in the early 1800 s, the species has undergone severe reductions in population size and number, and is currently listed as endangered under the national Environmental Protection and Biodiversity
Conservation Act 1999. Its known original range covered three main areas, each with breaks in distribution of approximately $500 \mathrm{~km}$ : the Canberra region in Australian Capital Territory (ACT) and New South Wales (NSW), west of Melbourne in Victoria (VIC), and on the Gippsland Plains in the east of VIC where it is now extinct (Morgan, 1995; Young et al., 1999). Highly fragmented populations now persist in habitat protected from farming and urbanisation (Scarlett \& Parsons, 1990). The current range of $R$. leptorrhynchoides comprises approximately 31 small disjunct natural populations, almost half of which contain $<200$ individuals, and none exceeding 100,000 (NSW OEH 2012 and subsequent unpublished census data from regional agencies). In an attempt to stem population decline, management actions have been undertaken including habitat restoration, monitoring and supplementary planting.

Previous research using nine allozyme loci (for 551 individuals) and ten microsatellites (for 364 individuals) showed differentiation among sampled populations of only $F_{\mathrm{ST}}=0.17$ for allozymes and $0.03-0.14$ for microsatellites, despite large breaks in the species' distribution. This was interpreted as suggesting a high degree of gene flow at least until recently (Young et al., 1999; Pickup et al., 2012). The lack of a major genetic break in neutral markers matching the large geographic disjunction is not unique in the literature for plant taxa with similar distributions in southeastern Australia such as Swainsona recta and Senecio macrocarpus (Buza et al., 2000; Ahrens et al., 2015). However, lack of strong population genetic subdivision could represent historical (pre-fragmentation) rather than contemporary genetic connectivity (Young et al., 1999), so population genetic datasets with greater resolution are required to assess gene flow. Genetic management of $R$. leptorrhynchoides has been proposed as part of its recovery plan (National Recovery Plan 2012). Current recommendations are to prioritise conservation of large, genetically diverse populations, because these will likely be the best sources for genetic rescue of smaller, less genetically diverse populations from similar environments, as supported by extensive crossing experiments including in the wild (Young et al., 1999; Pickup \& Young, 2008; Pickup et al., 2012, 2013). A detailed understanding of which populations are the most genetically diverse and which populations are most vulnerable is needed for targeted management. Furthermore, insights into the distribution of genetic variation and evolutionary processes such as past divergence and gene flow are necessary for defining populations for conservation prioritisation (Liddell et al., 2020).

In this study we use a dataset of 12,965 single nucleotide polymorphism (SNP) loci to investigate the evolutionary history of $R$. leptorrhynchoides and describe patterns of genome-wide diversity and structure in populations across 
its distribution. We aimed to: (1) quantify levels of genomewide differentiation between sampling locations and explore the underlying processes driving this differentiation; (2) assess levels of genome-wide genetic diversity within sampling locations and test whether they accord with previously published allozyme and microsatellite-based estimates; (3) infer some key parameters of population genetic history: population tree topology, divergence times and effective population sizes (scaled by mutation rate) were estimated by a coalescent tree method based on SNPs, and the role of gene flow in population isolation or admixture was explored via a maximum likelihood population tree based on SNP allele frequencies; and (4) use these results to define populations for conservation management planning. The molecular approaches we apply are likely to be affordable in many management scenarios, can be outsourced, do not require existing genome resources and hence are applicable to a wide range of species.

\section{Materials and methods}

\section{Study species}

Rutidosis leptorrhynchoides is a perennial herb endemic to south-eastern Australia. It occurs in grassy eucalypt woodlands and treeless grasslands, most of which are dominated by kangaroo-grass, Themeda triandra (Morgan, 1995). The species has a lifespan of greater than 10 years in the field and is pollinated primarily by insects of modest flight distance such as Lasioglossum bees (Morgan, 1995; Courtice et al., 2020). Seeds are weakly wind-dispersed, most falling close to the parent plant $(<50 \mathrm{~cm})$ and persisting for only a short time in the soil seed-bank (Morgan, 1995). The lack of regular long-distance robust dispersal is evidenced by the fine-scale spatial genetic structure (Wells \& Young, 2002). Rutidosis leptorrhynchoides has a single-locus sporophytic self-incompatibility system, in which pollen is identified as incompatible by the stigma and rejected when the pollen parent genotype at the $S$-locus carries alleles that match those at the $S$-locus receiving plant (Young et al., 2000; Mable et al., 2005). Reduction in $S$-allele diversity is detrimental for population fitness (Young \& Pickup, 2010). The species exhibits variation in chromosome number across its distribution, with northern and easternmost southern populations being diploid $(2 n=22)$ and majority of southern populations being autotetraploid, often with additional non-diploid karyotypes (Young et al., 1999). Here we focus solely on diploid populations, of which only two natural ones are known to remain in VIC in southern Australia, and $\sim 500 \mathrm{~km}$ to the north there are 11 in ACT and 10 in NSW (Fig. 1). The tetraploid/non-diploid populations, not considered here, occur in a coherent geographic range in
VIC, $>70 \mathrm{~km}$ west from the last two known diploid populations in the region. Mixed-ploidy crosses are possible but rare in nature, resulting in offspring with triploid and aberrant chromosome numbers, which are predominantly sterile (Brown \& Young, 2000; Young \& Murray, 2000; Murray \& Young, 2001). We therefore treat diploid populations as a discrete genetic management unit, separate from polyploid populations.

\section{Sampling, genotyping and SNP data filtering}

Leaf material was collected from a total of 199 individuals from 12 locations (10 ACT/NSW, 2 VIC) across the current distribution of diploid R. leptorrhynchoides in 2017/2018 (Fig. 1 and Table 1). Where practical, we sampled every population using a standard layout, defined by a rectangle measuring $20 \times 15 \mathrm{~m}$, containing 20 sample points in three rows, spaced at $5 \mathrm{~m}$ intervals: a row at $0,5,10,15,20 \mathrm{~m}$ on the long axis, repeated at $0,5,10,15 \mathrm{~m}$ on the short axis. We sampled genetic material from the plant closest to each point, avoiding new recruits judged $<1$ year old. Some sampling locations (BB, CF, CH, MI, SA) had plants over too small an area to accommodate this grid, so we sampled from across the population, as close to the $5 \mathrm{~m}$ grid arrangement as we could. Two populations (MI, SA) were so small numerically (13 and 18 individuals, respectively, Table 1) that we sampled from every plant we could locate on the day of sampling. One population $(\mathrm{CH})$ was widely dispersed in many small clusters, and to maximize consistency with the other samples, we sampled all plants from the largest cluster. For one location (TR) that is a particular focus for managers, in addition to the standard layout, we collected samples from plants around the population periphery.

Leaf samples were dried and sent to Diversity Arrays Technology Pty Ltd for DNA extraction and genotyping using the DArTseq ${ }^{\mathrm{TM}}$ (reduced-genome representation) platform (Jaccoud et al., 2001). DArTseq ${ }^{\mathrm{TM}}$ is similar to double-digest restriction-associated sequencing with the workflow optimised to lower rates of missing data, and a quarter of samples being re-analysed starting from library preparation step. Each locus is given a repeatability score based on sequencing of different libraries for the same samples, providing a basis for selecting markers with very low error rates (Georges et al., 2018). More details of DArTseq $^{\mathrm{TM}}$ can be found in Supplementary Methods S1.

The raw genomic dataset comprised 53,758 codominant, genome-wide, biallelic SNPs. Data filtering was done using the DARTR package (Gruber et al., 2018) in $R$ v3.5.0 (R Core Team, 2018). We removed 25,288 loci that were not $100 \%$ reproducible. With this selection criterion, nearly all low-frequency alleles should be reliable. In addition, we removed 11,779 loci with $>25 \%$ missing data per locus and 

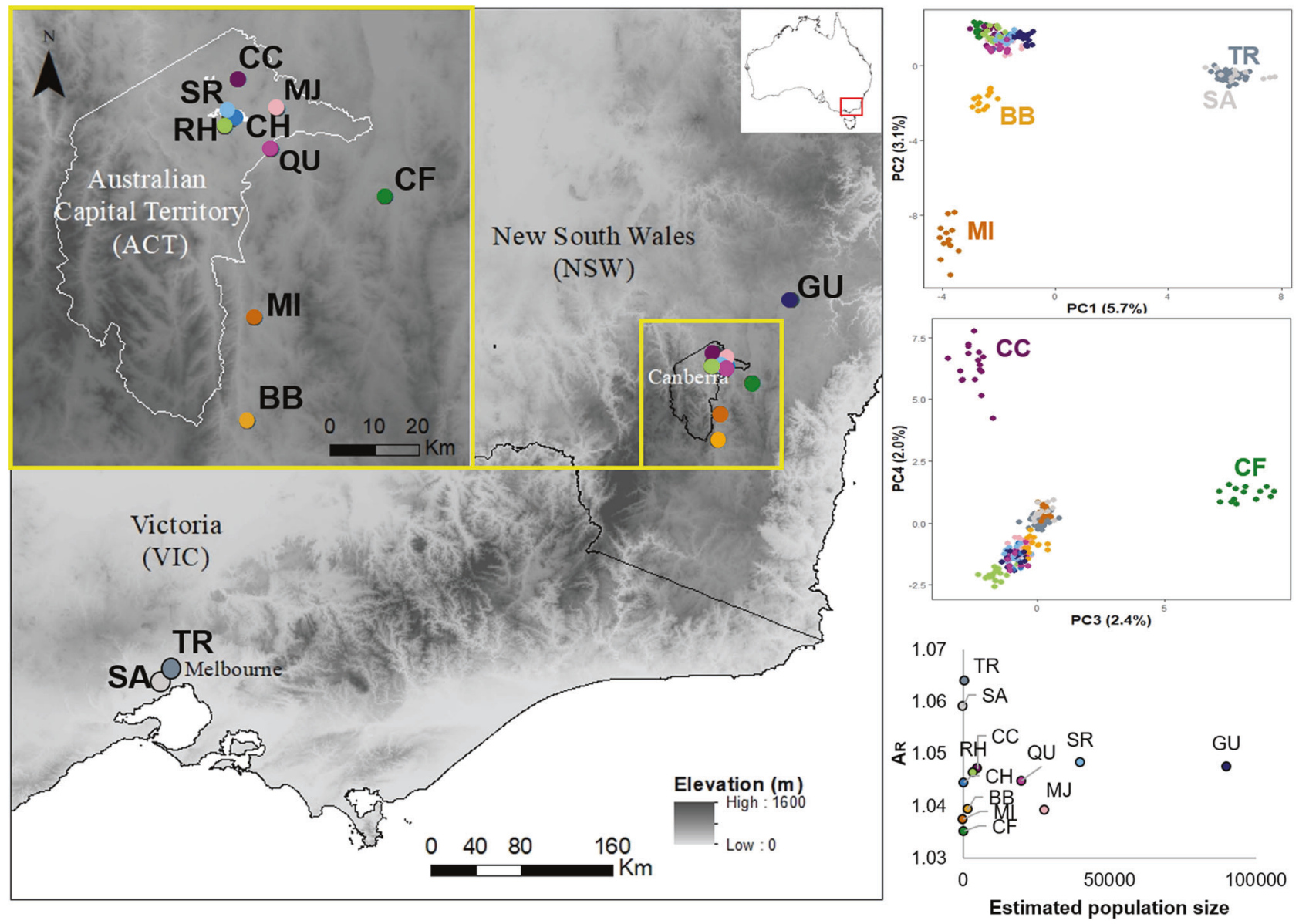

Fig. 1 Distribution of sampling locations for $\boldsymbol{R}$. leptorrhynchoides. Principal coordinate analysis (PCoA) derived from 12,965 SNPs for all 12 sampled populations. Population codes correspond to those presented in Table 1.

Table 1 List of sampling locations, state/territory, sample size $(N)$, estimated population size from the National Recovery Plan 2012.

\begin{tabular}{lllllll}
\hline Location & Code & State/territory & $N$ & Latitude & Longitude & Census size \\
\hline Gundary & GU & NSW & 20 & -34.8 & 149.74 & $\sim 90,000$ \\
Crace & CC & ACT & 17 & -35.23 & 149.13 & $\sim 5000$ \\
Majura & MJ & ACT & 17 & -35.29 & 149.21 & $\sim 28,000$ \\
Stirling Ridge & SR & ACT & 17 & -35.3 & 149.12 & $\sim 40,000$ \\
Capital Hill & CH & ACT & 6 & -35.31 & 149.12 & 293 \\
Red Hill & RH & ACT & 15 & -5.32 & 149.11 & $\sim 3440$ \\
Queanbeyan & QU & ACT & 15 & -35.37 & 149.2 & $\sim 20,000$ \\
Captains Flat & CF & NSW & 14 & -35.46 & 149.43 & 306 \\
Michelago & MI & NSW & 13 & -35.7 & 149.17 & 13 \\
Bredbo & BB & NSW & 14 & -35.91 & 149.15 & 1694 \\
Truganina & TR & VIC & 39 & -37.83 & 144.72 & $591^{\mathrm{a}}$ \\
St Albans & SA & VIC & 12 & -37.74 & 144.8 & $18^{\mathrm{a}}$ \\
Total & & & 199 & & &
\end{tabular}

Locations are shown in Fig. 1.

${ }^{\mathrm{a}}$ Recent census counts.

$51 F_{\mathrm{ST}}$ outliers (see below), and 4 individuals with $>15 \%$ missing data (along with 35 monomorphic loci associated with them). To control for very close physical linkage, we retained only one SNP per sequenced fragment ( $69 \mathrm{bp}$ ), which removed a further 3640 loci. We also filtered for monomorphic loci, but found none. 
To identify significant $F_{\mathrm{ST}}$ outliers, which could have evolved under selection and are likely to violate a Wright-Fisher model assumed in many approaches used, we ran BAYESCAN v. 2.1 (Foll \& Gaggiotti, 2008). The analysis was performed with 20 pilot runs each consisting of 5000 iterations, followed by 100,000 iterations with a 50,000 burn-in and a sample size of 5000. Prior odds for the neutral model were set to 100 , and the $F_{\text {IS }}$ prior was set to 'uniform between 0.0 and 0.4 ' based on the range of typical values for this inbreeding statistic for R. leptorrhynchoides (Pickup et al., 2012). The false discovery rate threshold for outlier locus detection was set at 0.05 . We found 51 significant outliers that were removed from the dataset.

The final filtered SNP dataset used for analyses contained 12,965 high-quality biallelic SNP loci with only $7.62 \%$ missing data. A second dataset of 1889 SNP loci with no missing data was also created for use in some analyses (below).

\section{Analysis of population genetic differentiation}

We inferred population structure by estimating the likely number of genetic clusters $(K)$ and membership of each individual in each genetic cluster using STRUCTURE v2.3.4 (Pritchard et al., 2000). To preclude inferences influenced by missing data, STRUCTURE was run using the 1889 SNP dataset with no missing data. Ten independent replicate runs were performed for each of $K=1-12$ genetic clusters (which would have been increased if warranted by the higher of these $K$ estimates), each with a burn-in of 500,000 followed by 1,000,000 Markov chain Monte Carlo (MCMC) iterations. We used the admixture model without prior population information. To identify further patterns of substructure, we performed additional STRUCTURE runs on only northern (ACT/NSW) populations, which belonged to a single cluster based on $K=2$ analysis, testing a range of genetic clusters from $K=1$ to 10 (Janes et al., 2017). CLUMPAK software (Kopelman et al., 2015) was used to summarise and visualise STRUCTURE output and perform the Evanno Delta $K$ method (Evanno et al., 2005) and $\ln \operatorname{Pr}(X \mid K)$ method of Pritchard \& Wen (2003) of determining best $K$. Based on the recommendations of Janes et al. (2017), we assessed the population structure and number of genetic clusters by applying and comparing these two methods. We checked that conclusions did not differ materially when using the full dataset in fastSTRUCTURE (Raj et al., 2014), and they did not (justification in Supplementary Figs. S11-S14).

To quantify genetic differentiation among populations, we estimated pairwise Weir \& Cockerham (1984) $F_{\mathrm{ST}}$ in the $R$ package HIERFSTAT (Goudet, 2005) using the full 12,965 loci dataset. In addition, a hierarchical analysis of molecular variance (AMOVA) was conducted in $R$ using the 'poppr.amova' function of POPPR (Kamvar et al., 2015), with significance testing using 999 permutations.
Populations were grouped into geographic regions: VIC (consisting of TR and SA) and ACT/NSW (consisting of $\mathrm{BB}, \mathrm{CC}, \mathrm{CH}, \mathrm{CF}, \mathrm{GU}, \mathrm{MI}, \mathrm{MJ}, \mathrm{QU}, \mathrm{RH}$ and SR). Principal coordinate analysis ( $\mathrm{PCoA}$ ) was performed in the $R$ package DARTR (Gruber et al., 2018). PCoA analysis was also performed on the no-missing dataset and yielded similar results (Supplementary Fig. S15). We tested for an isolation by distance model of differentiation by plotting geographic distance versus $F_{\mathrm{ST}} /\left(1-F_{\mathrm{ST}}\right)$ and performing a Mantel test using the function 'gl.ibd' in DARTR for all 12 populations and for populations only within the ACT/NSW region.

\section{Estimation of within-population diversity}

Observed heterozygosity $\left(H_{\mathrm{o}}\right)$, gene diversity $\left(H_{\mathrm{S}}\right)$, allelic richness $\left(A_{R}\right)$ and $F_{\text {IS }}$ for each population were estimated using HIERFSTAT. Genetic diversity analyses were also performed on the no-missing dataset and yielded similar results (Supplementary Fig. S16). To gain some insight into how different the VIC and ACT/NSW populations are genetically, we estimated the frequency of private alleles per region using the $\mathrm{R}$ package POPPR (Kamvar et al., 2015). Individuals were grouped into their respective regions (VIC or ACT/NSW) and the frequency distributions of alleles unique to each region were assessed to determine whether the majority of unique alleles are rare or common within each sample.

A correlation between low diversity and high populationspecific $F_{\mathrm{ST}}$ indicates that loss of diversity through genetic drift drives apparent differentiation (Coleman et al., 2013). To test for evidence of drift in our data, we calculated mean population-specific $F_{\mathrm{ST}}$ for each population in GESTE v. 2.0 (Foll \& Gaggiotti, 2006) and performed a simple linear regression against genetic diversity $\left(A_{R}\right.$ and $\left.H_{\mathrm{o}}\right)$.

\section{Population history and connectivity}

To explore history of population divergence, we inferred a population tree using a coalescent model and Bayesian MCMC approach implemented in SNAPP (Bryant et al., 2012) run using BEAST 2.6 (Bouckaert et al., 2019). SNAPP's model builds coalescent trees for each SNP locus independently and then integrates over all possible genealogies to provide estimates of tree topology and parameters of population divergence times and effective population sizes, scaled by mutation rate. The SNAPP model assumes divergence without gene flow among genetic populations. For SNAPP analyses to complete in feasible computational times, we used reduced SNP datasets of 2000 loci randomly chosen from the full 12,965 loci dataset and four randomly selected individuals to represent each population (Spalink et al., 2019; Rojas et al., 2020). We used the default prior and model parameters $(\mu=1, v=1$, coalescence rate $=10$, priors: $\alpha=11.75, \beta=109.73, \kappa=1, \lambda=0.00765)$ and ran 
two independent replicate MCMC runs of 1,000,000 iterations with sampling every 1000 steps and a burn-in of $10 \%$. To ensure the choice of individuals and loci did not influence our results, this analysis was repeated on another set of randomly sampled individuals and 2000 randomly sampled loci.

We used TRACER v. 1.7.1 (Rambaut et al., 2018) to visually inspect the output for acceptable mixing and replicate run convergence, confirming effective sample sizes $>200$ for all parameters. The two runs for each replicate were combined using LOGCOMBINER (Drummond \& Rambaut, 2007), using a burn-in of $10 \%$. The posterior distribution of gene (SNP) trees was visualised using DENSITREE v. 2.6.1 (Bouckaert, 2010) and summarised by generating a maximum clade credibility (MCC) tree using TREEANNOTATOR v. 2.6 (Drummond \& Rambaut, 2007).

To test whether historical or contemporary migration resulting in gene flow could explain variation in addition to that explained by populations diverging in isolation, and to reveal potential admixture events, we used TreeMix v1.13 (Pickrell \& Pritchard, 2012) on the full 12,965 SNP dataset. TreeMix uses allele frequency data to approximate an unrooted maximum likelihood population tree. A stepwise likelihood procedure is used to test the effect of migration on the residual covariance matrix and determine the optimal placement of migration events in the population tree. We inferred a topology without admixture, as well as allowing up to five migration events.

\section{Results}

\section{Levels of differentiation and diversity in $R$. leptorrhynchoides}

Pronounced genetic differentiation between VIC versus ACT/NSW sampling locations for R. leptorrhynchoides was supported by several different analyses. The first axis (PC1) of the PCoA analysis explained $6.17 \%$ of the total variance and clearly separated VIC locations from ACT/NSW (Fig. 1). Additional axes, PC2, PC3 and PC4, separated out ACT/NSW sampling locations, accounting for $3.05 \%, 2.4 \%$ and $1.9 \%$ of the total variation, respectively. The NSW locations $\mathrm{BB}$ and $\mathrm{MI}$ were the next most differentiated from the rest of the northern ones according to PC2 (Fig. 1). No clear differentiation was observed between the two VIC locations across the first six PC axes (Supplementary Fig. S1).

In the STRUCTURE analysis on all 12 sampling locations, the optimal value of $K$ was 2 according to the Delta $K$ method, which usually reflects the top level of structure in the data (Janes et al., 2017) (Supplementary Fig. S2), with VIC forming one cluster and ACT/NSW a second. Although output for $K>2$ consistently separated southern (VIC) and northern (ACT/NSW) locations, the runs were insufficiently consistent to determine a best $K$ according to $\ln \operatorname{Pr}(X \mid K)$ method (Supplementary Fig. S3). In the analysis of ACT/NSW alone, the Delta $K$ method supported the presence of nine genetic clusters, and the $\ln \operatorname{Pr}(X \mid K)$ method supported eight (Supplementary Fig. S4). With $K=8-10$, almost all sampling locations were assigned to their own cluster, with the exceptions that $\mathrm{SR}$ and $\mathrm{CH},<1 \mathrm{~km}$ apart, were assigned to the same cluster (Fig. 2; for all ACT/NSW STRUCTURE outputs refer to Supplementary Fig. S5).

When sampling locations were grouped into geographic regions, AMOVA revealed that grouping sampling locations into the north and south regions explained $8.08 \%$ of variance $\left(\phi_{\mathrm{CT}}=0.081, p=0.001\right)$, and sampling locations within regions explained $21.50 \%\left(\phi_{\mathrm{SC}}=0.234, p=0.001\right)$. Similarly, pairwise $F_{\text {ST }}$ comparisons showed significant pairwise differentiation among most sampled locations, with largest values observed for comparisons involving MI and CF in NSW, and SA in VIC (Table 2). No significant isolation by distance was detected among all locations $(r=0.294, p=0.109$, Supplementary Fig. S6a) or among those in ACT/NSW alone $(r=0.481, p=0.057$, Supplementary Fig. S6b).

a)

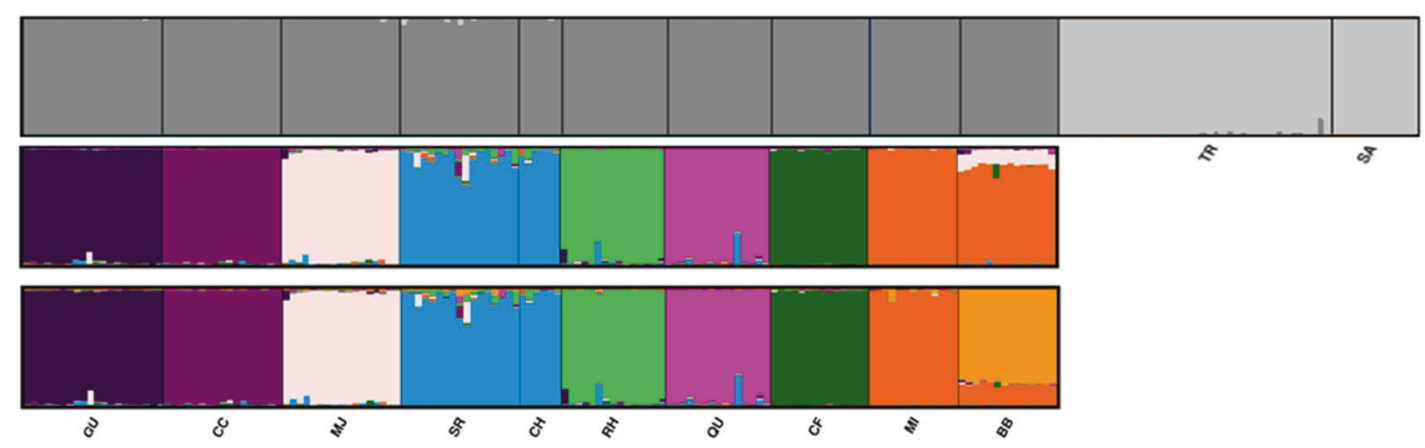

Fig. 2 STRUCTURE clustering of $R$. leptorrhynchoides individuals based on 1889 SNPS. Plots for a all 12 populations with an optimum $K$ value of 2, b ACT/NSW populations only (which formed part of a single genetic cluster when $K=2$ for all 12 populations) for $K=8$,

and c $K=9$. Each vertical bar corresponds to an individual and the vertical axis is membership ( $Q$ value) in each of the $K$ genetic clusters. Population codes correspond to those presented in Table 1. 
Genetic diversity and population size estimates (Table 1) were not correlated $\left(r^{2}=0.007, p=0.80\right.$, Fig. 1 and Supplementary Fig. S7). The VIC locations TR and SA had the highest heterozygosities (Fig. 3). In contrast, comparably small populations at the southern end of the northern range - BB, CF and $\mathrm{MI}$ - had the lowest levels of genetic diversity by all measures. It is also notable that MJ had one of the lowest $H_{\mathrm{O}}(0.021)$ and relatively low $A_{\mathrm{R}}(1.14)$ even though it is one of the larger populations, estimated to number $>28,000$ plants (Table 1, Fig. 1 and Supplementary Fig. S7).
The majority $(62.0 \%)$ of the 25,930 alleles in the full dataset were shared in common between samples from ACT/NSW and those from VIC. While $19.8 \%$ of the 25,930 were present only in ACT/NSW, and $18.3 \%$ were unique to $\mathrm{VIC}$, most of these private alleles were rare, representing $<5 \%$ of the sample for a given marker (Supplementary Fig. S8). Some very rare alleles may not have been adequately detected with the sample size of 51 individuals in VIC but alleles $>5 \%$ in the population should have been detected $>95 \%$ of the time (Sjogren \& Wyoni, 1994).
Table 2 Pairwise $F_{\mathrm{ST}}$ values among sampled populations of $R$. leptorrhynchoides based on 12,965 SNPs.

\begin{tabular}{llllllllllll}
\hline Population & GU & CC & MJ & SR & CH & RH & QU & CF & MI & BB & TR \\
\hline CC & 0.13 & & & & & & & & & \\
MJ & 0.1 & 0.13 & & & & & & & & \\
SR & 0.08 & 0.11 & 0.080 & & & & & & & & \\
CH & 0.11 & 0.14 & 0.13 & 0.05 & & & & & & & \\
RH & 0.11 & $\mathbf{0 . 1 4}$ & 0.12 & 0.08 & 0.11 & & & & & & \\
QU & 0.110 & $\mathbf{0 . 1 4}$ & 0.11 & $\mathbf{0 . 0 9}$ & $\mathbf{0 . 1 2}$ & $\mathbf{0 . 1 2}$ & & & & \\
CF & $\mathbf{0 . 1 9}$ & $\mathbf{0 . 2 2}$ & $\mathbf{0 . 2 1}$ & $\mathbf{0 . 1 7}$ & $\mathbf{0 . 2 2}$ & $\mathbf{0 . 2 1 0}$ & 0.2 & & & \\
MI & $\mathbf{0 . 2 3}$ & $\mathbf{0 . 2 5}$ & $\mathbf{0 . 2 5}$ & $\mathbf{0 . 2 2}$ & $\mathbf{0 . 2 6}$ & $\mathbf{0 . 2 5}$ & $\mathbf{0 . 2 4}$ & $\mathbf{0 . 3 2}$ & & & \\
BB & $\mathbf{0 . 1 4}$ & $\mathbf{0 . 1 7}$ & $\mathbf{0 . 1 5 0}$ & $\mathbf{0 . 1 2}$ & $\mathbf{0 . 1 7}$ & $\mathbf{0 . 1 6}$ & 0.15 & $\mathbf{0 . 2 4 0}$ & $\mathbf{0 . 2 3}$ & & \\
TR & $\mathbf{0 . 1 2}$ & $\mathbf{0 . 1 5}$ & $\mathbf{0 . 1 3}$ & $\mathbf{0 . 1 1}$ & $\mathbf{0 . 1 2}$ & $\mathbf{0 . 1 4}$ & $\mathbf{0 . 1 3}$ & $\mathbf{0 . 1 9}$ & 0.22 & 0.16 & \\
SA & $\mathbf{0 . 1 6}$ & $\mathbf{0 . 2}$ & $\mathbf{0 . 1 9}$ & $\mathbf{0 . 1 6}$ & $\mathbf{0 . 1 6}$ & $\mathbf{0 . 1 9}$ & $\mathbf{0 . 1 8}$ & $\mathbf{0 . 2 6}$ & $\mathbf{0 . 2 9}$ & $\mathbf{0 . 2 1}$ & $\mathbf{0 . 0 4}$ \\
\hline B & &
\end{tabular}

Bold values are significant $(p<0.05)$. Population codes correspond to those presented in Table 1 .

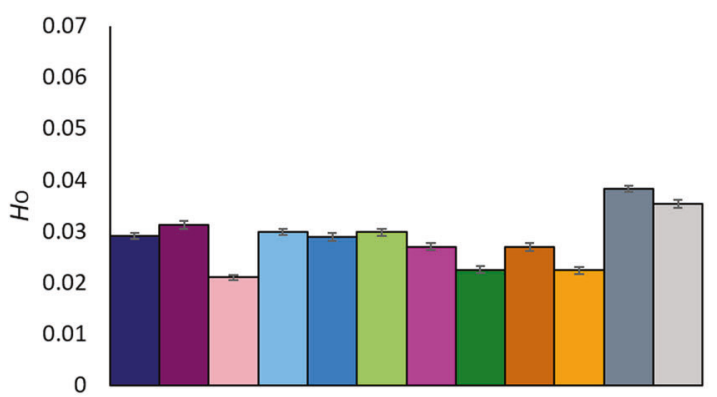

GU CC MJ SR CH RH QU CF MI BB TR SA

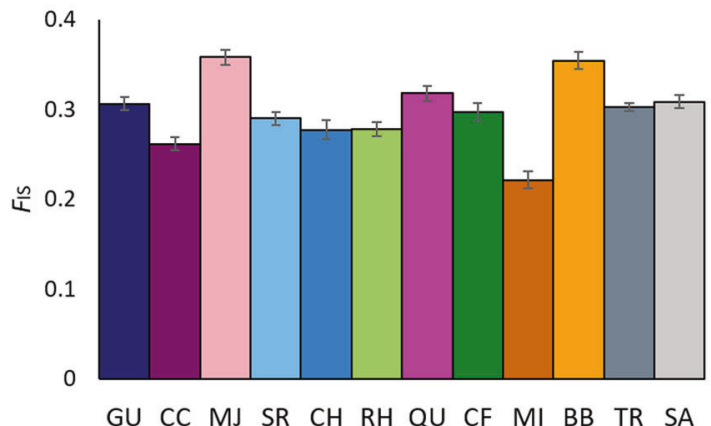

Fig. 3 Summary of genetic diversity parameters for $R$. leptorrhynchoides populations sampled in this study, estimated from 12,965 loci. Population codes correspond to those presented in

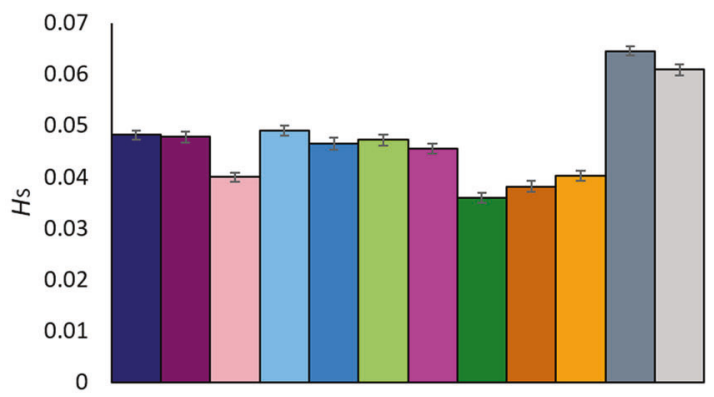

GU CC MJ SR CH RH QU CF MI BB TR SA

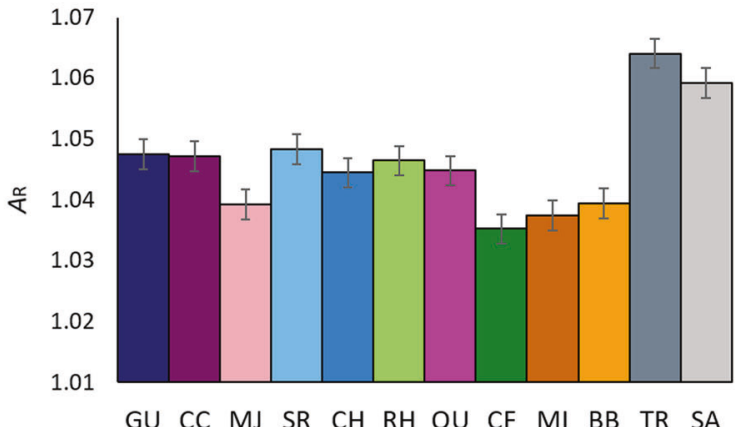

Table $1 . H_{\mathrm{o}}$ observed heterozygosity, $H_{\mathrm{S}}$ gene diversity, $A_{R}$ allelic richness. Numerical values are presented in Supplementary Table S3. 


\section{Divergence between genetic populations is consistent with genetic drift in isolation}

There was strong support for a genetic split between VIC (TR and SA) and all northern locations, according to the SNAPP MCC population trees produced by the two different datasets (posterior probability $\mathrm{PP}=1$ for VIC and ACT/NSW branches, Fig. 4 and Supplementary Fig. S9). All clades that showed $\mathrm{PP}>0.75$ were supported by both datasets. $\mathrm{CF}$ is shown as an ancestral population of the rest of ACT/NSW populations, which themselves form a well-supported clade $(\mathrm{PP}=1)$. The grouping together of the two locations in the southern part of the species distribution in NSW (BB and MI) also received high support $(\mathrm{PP}=1)$, as did the two sites in ACT that are near each other, $\mathrm{SR}$ and $\mathrm{CH}(\mathrm{PP}=1)$. Relationships between $\mathrm{GU}$ and the cluster of ACT populations (all of the latter are in close geographic proximity) are not well resolved, likely due to past gene flow. Estimates of average effective population size over time scaled by mutation rate $(\theta)$ were highly correlated with estimates of genetic diversity per population (Supplementary Table S3 and Supplementary Fig. S18).

The unrooted maximum likelihood tree inferred by TreeMix (with no migration events added) showed topology largely concordant with population clustering inferred by the PCoA, STRUCTURE and SNAPP analyses (Fig. 5). The two VIC locations are grouped together, divergent from ACT/NSW, and CF, MI and BB in NSW are grouped close

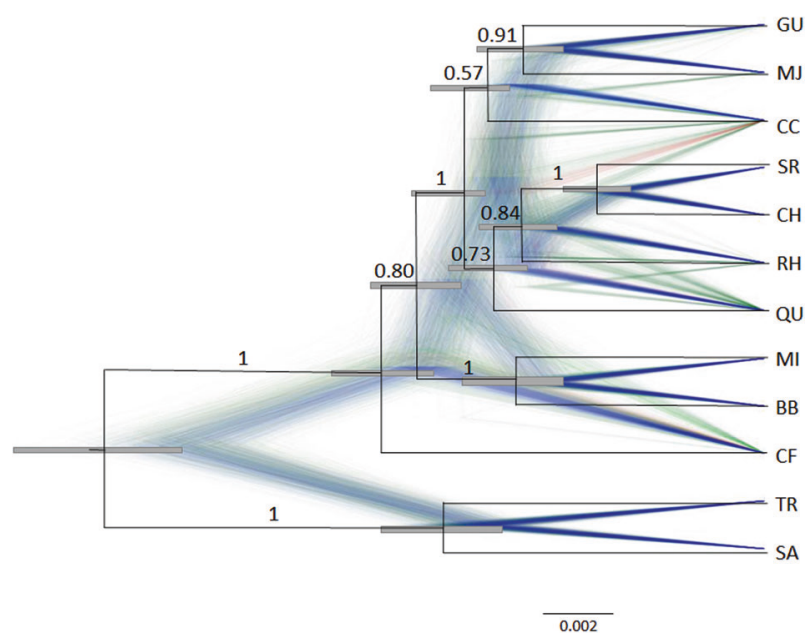

Fig. 4 Combined SNAPP population tree of $R$. leptorrhynchoides populations based on 2000 SNPs. Maximum clade credibility tree generated in FigTree v. 1.4.4 (https://github.com/rambaut/figtree/releases) is in black with 95\% HPD for height at each node indicated by the grey bar. Branch lengths are measured in expected substitutions per site. Densitree of superimposed gene trees recorded during the MCMC analysis visualises the range of alternative topologies, indicative of past gene flow. Gene trees shown in blue lines indicate most frequent trees, next most frequent are red, third most are green. Numbers represent posterior probabilities of branches. Population codes correspond to those presented in Table 1. together and show high levels of drift, as indicated by the length of horizontal branches, which are proportional to the amount of genetic drift that has occurred since a population became isolated. The inference of strong drift processes was supported by the population-specific $F_{\mathrm{ST}}$ analysis, as follows. Populations that were more genetically distinct had lower genetic diversity: regression of the mean populationspecific $F_{\mathrm{ST}}$ against $H_{\mathrm{O}}$ and $A_{\mathrm{R}}$ yielded strong negative relationships $\left(R^{2}=0.655, p=0.001\right.$, and $R^{2}=0.882, p<$ 0.001 , respectively, Fig. 6), suggesting that drift drives the apparent differentiation in populations.

The strongly positive TreeMix residuals among some pairs of locations indicate that these may be more closely related than they appear in the tree, and are thus candidates for potential admixture events (Fig. 5). Tree topology did not change significantly with the addition of subsequent migration events, although the fit of the data was optimised with three migration edges (Supplementary Fig. S10). The low weights of these proposed migration events are related to the low proportion of alleles in the descendent populations that are derived from the ancestral population and may be reflective of past gene flow (Pickrell \& Pritchard, 2012). Past gene flow between GU and CC is supported by their close grouping and the low support for their split in the SNAPP tree ( $\mathrm{PP}=0.57$, Fig. 4).

\section{Discussion}

\section{Major genetic split between northern and southern populations reflects geography}

We found clear genetic differentiation between the southern (VIC) and northern (ACT/NSW) populations of R. leptorrhynchoides, which was hinted at but not resolved in
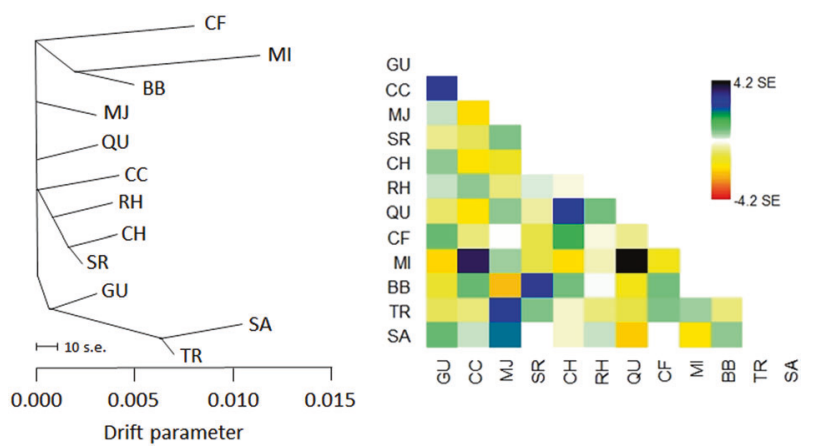

Fig. 5 TreeMix analysis of $R$. leptorrhynchoides based on 12,965 SNPs showing the unrooted maximum likelihood tree, and the residual fit from the tree. Drift parameter is shown on the $x$-axis and the scale bar shows 10 times the average standard error of the entries in the sample covariance matrix. Large, positive residuals (blue-black colours) indicate population pairs that are more closely related to each other in the data than suggested by the best-fit tree, and may be candidates for admixture events. 

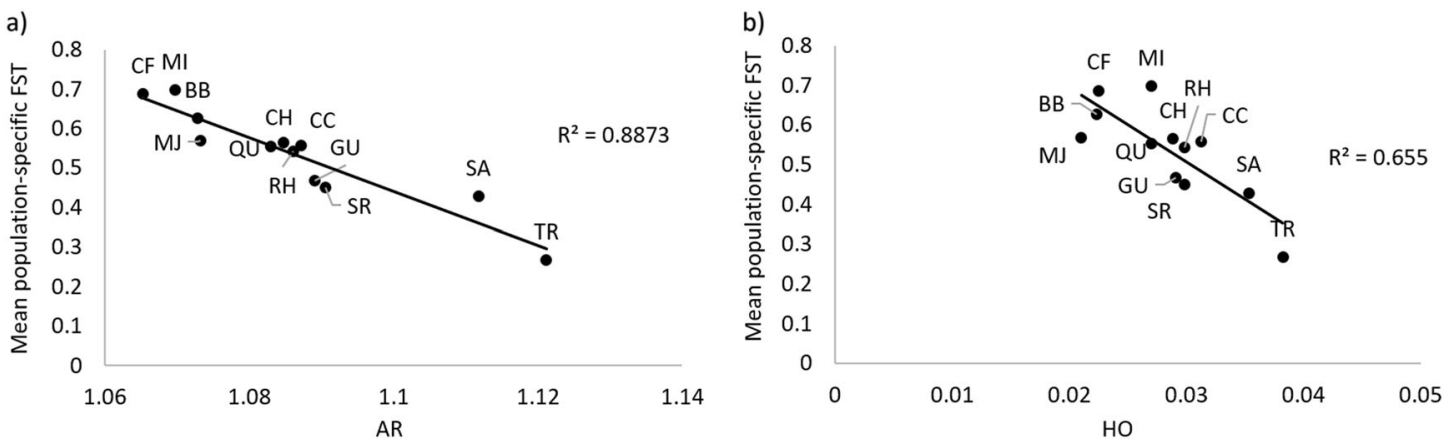

Fig. 6 Regressions between mean population-specific $\boldsymbol{F}_{\mathrm{ST}}$ and estimates of genetic diversity using 12,965 SNPs. a Allelic richness $A \mathrm{R}$ vs mean population-specific $F_{\mathrm{ST}}$ and $\mathbf{b}$ observed heterozygosity $\mathrm{HO}$ vs mean population-specific $F_{\mathrm{ST}}$.

previous population genetic studies of this species (Pickup et al., 2012, 2013). This probably reflects the relatively low resolution of the techniques previously available, and highlights the value of genome-wide SNPs for improving our understanding of fine population genetic structure. The observed genetic split reflects the break in the current geographic distribution of the species. This discontinuity in distribution is mirrored by many other southern Australian species native to grassy woodlands and grasslands dominated by tussock-forming grasses, including plants (Department of the Environment, 2020; Buza et al., 2000; Sinclair, 2010), insects (Clarke \& O'Dwyer, 2000) and reptiles (Dorrough \& Ash, 1999; Melville et al., 2007), many of which are of high conservation concern. For example, a phylogenetic study of VIC and ACT/NSW populations of the grassland earless dragon ( $T$. pinguicolla) found major genetic structure between the regions, dating back to the late Pliocene (Melville et al., 2007).

Large-scale connection and disconnection of grassland habitats across south-eastern Australia, driven by changing climate, were likely drivers of these biogeographical patterns. Periods of forest expansion and contraction and marine incursions in near-coastal part of the species' range during late Miocene to mid-Pleistocene (0.7-5 Ma) glacial oscillations likely repeatedly caused $R$. leptorrhynchoides distribution to contract to the grassy woodlands of ACT and the grassy plains of VIC, promoting divergence (Bowler, 1982; Markgraf et al., 1995; Jones, 1997). Few isolated patches may have remained outside these strongholds, such as the one recorded in the Gippsland Plains of eastern VIC (Young et al., 1999). Because the vast majority of intervening forest habitat is currently unsuitable for the species, and given the species' low dispersal ability (Morgan, 1995), gene flow between north and south likely ceased at the last such grassland contraction, possibly during the Holocene (last $\sim 10,000$ years). The onset of land conversion and grazing by exotic ungulates in the last $\sim 180$ years (Powell, 1969; Weaver, 1996) would have resulted in reduced, isolated populations within the centres of distribution
(ACT and the grassy plains of VIC), leading to the strong drift inferred here.

\section{Isolation and differentiation of populations with genetic drift}

Similar to other outcrossing plant species (Nybom \& Bartish, 2000), differences among individuals within populations accounted for the majority of variation in $R$. leptorrhynchoides. Our data implicate genetic drift in isolation as the driving force behind this differentiation, particularly within the southern NSW locations of CF, BB and MI. CF was previously identified as isolated, using STRUCTURE analysis (Pickup et al., 2012).

Loss of genetic diversity through drift is particularly detrimental in self-incompatible species, including $R$. leptorrhynchoides, owing to the loss of variation at the $S$-locus, which controls self-incompatibility (Pickup \& Young, 2008; Young \& Pickup, 2010). If the number of $S$-alleles falls too low for the breeding system to be sufficiently successful, small self-incompatible plant populations will be highly vulnerable to extinction (e.g., DeMauro, 1993; Wagenius et al., 2007), as supported for R. leptorrhynchoides through stochastic matrix projection models parameterised by data on growth, survival and reproduction of four life stages measured in multiple years for each population of interest (Young et al., 2000). Thus, small selfincompatible populations with low genetic diversity are highly likely to benefit from re-establishing gene flow (Frankham, 2015; Frankham et al., 2017 p. 128). Glasshouse and common garden experiments with $R$. leptorrhynchoides suggest that the species is a good candidate for genetic rescue, with evidence of increased seed set and heterosis following inter-population mixing, even between northern and southern genetic clusters (Pickup \& Young, 2008; Pickup et al., 2013). Re-establishing natural gene flow among isolated populations such as $\mathrm{CF}, \mathrm{BB}$ and $\mathrm{MI}$ may be challenging in the human impacted landscapes that drove their isolation and population contractions, given the 
species biology. However, at least in the short term, genetic restoration could be achieved relatively easily by in situ plantings.

\section{High genetic diversity retained in small Victorian populations}

Plant species with declining population sizes are expected to have lower genetic diversity due to drift within populations, compared to commoner and more-widespread species (Frankham, 1996; Cole, 2003). However, our data showed no correlation between estimated population size and genetic diversity (Fig. 1 and Supplementary Fig. S7). Despite their small and declining size, we found that $R$. leptorrhynchoides in VIC have so far retained high levels of genetic diversity relative to their population size, similar to the findings based on allozymes and microsatellites (Young et al., 1999; Pickup et al., 2012). In our data, TR (population size 591 plants) and SA (18) have the highest levels of heterozygosity and allelic richness, higher than those in populations of comparable size in ACT/NSW such as CF (306) and MI (13) as well as those of much larger size such as GU $(\sim 90,000)$ and MJ $(\sim 28,000)$. High genetic diversity and low differentiation in small VIC populations could reflect population reductions being too recent for loss of genetic diversity through drift (Young et al., 1999; Chen et al., 2017). TR and SA are located on the fringes of Melbourne city, are the last known survivors among populations lost since 1950 (Bull \& Stolfo, 2014) and have been subject to very recent decline (recent TR population size is $\sim 60 \%$ of its size of $>1000$ in 2002 and SA is $\sim 13 \%$ of the 137 reproductive individuals in 2000 , Brown \& Young, 2000). Together with this relatively recent bottleneck due to human habitat destruction, diversity is likely buffered by the relatively long generation time of $R$. leptorrhynchoides (7-15 years in the wild) compared to the duration of habitat destruction (regionally $<200$ years; locally decades), and relatively long longevity ( $>10$ years; Scarlett \& Parsons, 1990), as seen in other plant species (Lippé et al., 2006; Aægisdóttir et al., 2009). Selfincompatibility tends to act to retain genetic variation in populations by promoting outbreeding, which equalises family sizes, reduces genetic drift and promotes natural selection (Delph \& Kelly, 2014). The negative frequencydependent selection acting on $S$-alleles buffers rare alleles from loss and can act to maintain high diversity at nearby genomic regions (Glémin et al., 2005; Charlesworth, 2006). Although these processes will occur in all natural populations, they may have slowed loss of diversity of only very recently bottlenecked VIC populations but not some ACT/ NSW populations that have been small-and thus experienced strong drift and weak selection-for a longer time.

Presently, $R$. leptorrhynchoides is of high conservation concern in VIC, where recruitment is low. Given the ongoing decline and fragmentation of these populations, coupled with the limited dispersal ability of this species, it is likely that without intervention these populations will become differentiated through loss of diversity due to genetic drift as suggested by our data for some populations in the northern region.

\section{Conclusions and conservation implications}

Many northern (ACT/NSW) populations of $R$. leptorrhynchoides are declining and losing genetic diversity via drift, with measurable negative fitness effects (present data; Pickup \& Young, 2008). For example, isolation and low genetic variation of the small NSW populations of BB, MI and $\mathrm{CF}$ indicate that without intervention they are vulnerable to extinction. While such inbred populations are expected to gain fitness through gene flow from genetically diverse, differentiated populations of their region, in the absence of outbreeding depression, genetic rescue effects are predicted to be even greater using source populations that are more genetically variable and diverged from the recipients (Frankham, 2015; Frankham et al., 2017). Benefits can be substantial even from differently adapted populations (Kronenberger et al., 2017). Consistent with these expectations, breeding experiments in $R$. leptorrhynchoides showed only positive fitness consequences of crossing between northern and southern populations, and greater fitness gain when the source population had higher genetic diversity and lower inbreeding (Pickup \& Young, 2008; Pickup et al., 2012, 2013). Thus, the high levels of genetic diversity and unique variation in the small, isolated VIC populations of $R$. leptorrhynchoides highlight their potential value as a key resource for active genetic management of northern populations of this endangered species suffering genetic erosion. Ex situ conservation activities are ongoing for these populations and should remain a priority.

Due to their small and declining population sizes, VIC populations are vulnerable to extinction through environmental stochasticity, and would benefit from demographic rescue. The surprisingly high genetic diversity at TR and SA given their census sizes indicate that demographic augmentation may be of greater urgency than is elevating genetic diversity. Nonetheless, both locations will be losing genetic variation by drift, and SA in particular comprises so few individuals that low $S$-allele diversity may be limiting reproduction (Young et al., 2000). Both VIC populations are sufficiently small to hinder adaptation to new environments, and because they are genetically similar to each other, genetic augmentation from the warmer and drier north could also be considered as a means to help counter changing climate and weather (Frankham et al., 2017). Such decisions can be made by weighing up the risks of genetic 
erosion from not mixing gene pools versus any possible genetic harm from mixing them (Ralls et al., 2018; Liddell et al., 2020).

\section{Data availability}

DArT genotypes and associated geographic data for each individual are available at Bridges Monash University research repository https://doi.org/10.26180/5ea1603edd3a1. Raw DArT read data are at https://doi.org/10.26180/ 5ce65bf202a3e.

Acknowledgements For assistance with site information, sample collection, or access permission, we thank J. Morgan, J. Briggs, R. Armstrong, P. Palmer, A. Deans, J. Pittock, E. Barnett, B. Courtice, G. Evans, M. Gilbert, P. Beutel and P. Foreman. J. Morgan provided useful discussions about life history and ecology of $R$. leptorrhynchoides. This work was supported by an Australian Research Council Linkage Grant LP160100482 to Monash University and La Trobe University, with Partner Organisations University of Canberra, Department of Environment, Land, Water and Planning (DELWP, Victoria), Diversity Arrays Technology, Zoos Victoria, Environment, Planning \& Sustainable Development Directorate (ACT Government) and Department of Biodiversity, Conservation and Attractions (Western Australia). Additional support was provided by the Holsworth Wildlife Research Endowment and Parks Victoria's Research Partners Panel. Computationally intensive analyses were run on the Monash Computing Cluster courtesy of Monash eResearch. YSR was supported by the Monash Graduate Scholarship (MGS) and Dean's International Postgraduate Research Scholarship (DIPRS). We thank the Associate Editor and two anonymous reviewers for insightful comments that greatly improved this manuscript.

\section{Compliance with ethical standards}

Conflict of interest The authors declare no competing interests.

Publisher's note Springer Nature remains neutral with regard to jurisdictional claims in published maps and institutional affiliations.

Open Access This article is licensed under a Creative Commons Attribution 4.0 International License, which permits use, sharing, adaptation, distribution and reproduction in any medium or format, as long as you give appropriate credit to the original author(s) and the source, provide a link to the Creative Commons license, and indicate if changes were made. The images or other third party material in this article are included in the article's Creative Commons license, unless indicated otherwise in a credit line to the material. If material is not included in the article's Creative Commons license and your intended use is not permitted by statutory regulation or exceeds the permitted use, you will need to obtain permission directly from the copyright holder. To view a copy of this license, visit http://creativecommons. org/licenses/by/4.0/.

\section{References}

Aægisdóttir HH, Kuss P, Stöcklin J (2009) Isolated populations of a rare alpine plant show high genetic diversity and considerable population differentiation. Ann Bot 104:1313-1322

Ahrens CW, James EA, Botanic R, Melbourne G, Ave B, Yarra S (2015) Range-wide genetic analysis reveals limited structure and suggests asexual patterns in the rare forb Senecio macrocarpus. Biol J Linn Soc 115:256-269

Bouckaert R (2010) DensiTree: making sense of sets of phylogenetic trees. Bioinformatics 26:1372-137

Bouckaert R, Vaughan TG, Barido-Sottani J, Duchene S, Fourmet M, Gavryushkina A et al. (2019) BEAST 2.5: an advanced software platform for Bayesian evolutionary analysis. PLoS Comput Biol $15: 1-28$

Bowler J (1982) Aridity in the late tertiary and quaternary of Australia. In: Barker W, Greenslade P (eds) Evolution of the flora and fauna of arid Australia. Peacock Publications, Adelaide, p 35-45

Breed MF, Harrison PA, Blyth C, Byrne M, Gaget V, Gellie NJC et al. (2019) The potential of genomics for restoring ecosystems and biodiversity. Nat Rev Genet 20:615-628

Brown AHD, Young AG (2000) Genetic diversity in tetraploid populations of the endangered daisy Rutidosis leptorrhynchoides and implications for its conservation. Heredity (Edinb) 85:122-129

Bryant D, Bouckaert R, Felsenstein J, Rosenberg NA, Roychoudhury A (2012) Inferring species trees directly from biallelic genetic markers: bypassing gene trees in a full coalescent analysis. Mol Biol Evol 29:1917-1932

Bull M, Stolfo G (2014) Flora of Melbourne. A guide to the indigenous plants of the greater Melbourne area, 4th edn. Hyland House, Melbourne

Buza L, Young A, Thrall P (2000) Genetic erosion, inbreeding and reduced fitness in fragmented populations of the endangered tetraploid pea Swainsona recta. Biol Conserv 93:177-186

Charlesworth D (2006) Balancing selection and its effects on sequences in nearby genome regions. PLoS Genet 2:379-384

Chen C, Lu RS, Zhu SS, Tamaki I, Qiu YX (2017) Population structure and historical demography of Dipteronia dyeriana (Sapindaceae), an extremely narrow palaeoendemic plant from China: implications for conservation in a biodiversity hot spot. Heredity (Edinb) 119:95-106

Clarke GM, O'Dwyer C (2000) Genetic variability and population structure of the endangered golden sun moth, Synemon plana. Biol Conserv 92:371-381

Cole CT (2003) Genetic variation in rare and common plants. Annu Rev Ecol Evol Syst 34:213-237

Coleman RA, Weeks AR, Hoffmann AA (2013) Balancing genetic uniqueness and genetic variation in determining conservation and translocation strategies: a comprehensive case study of threatened dwarf galaxias, Galaxiella pusilla (Mack) (Pisces: Galaxiidae). Mol Ecol 22:1820-1835

Courtice B, Hoebee SE, Sinclair S, Morgan JW (2020) Local population density affects pollinator visitation in the endangered grassland daisy Rutidosis leptorhynchoides (Asteraceae). Aust J Bot 67:638-648

Crandall KA, Bininda-Emonds ORP, Mace GM, Wayne RK (2000) Considering evolutionary processes in conservation biology. TREE 15:290-295

Delph LF, Kelly JK (2014) On the importance of balancing selection in plants. N Phytol 201:45-56

DeMauro MM (1993) Relationship of breeding system to rarity in the Lakeside Daisy (Hymenoxys acaulis var. glabra). Conserv Biol $7: 542-550$

Department of the Environment (2020) Senecio macrocarpus in Species Profile and Threats Database, Department of the Environment, Canberra. Available from: http://www.environment.gov.au/ sprat. Accessed 27 May 2020.

Diekmann OE, Gouveia L, Perez JA, Gil-Rodriguez C, Serrão EA (2010) The possible origin of Zostera noltii in the Canary Islands and guidelines for restoration. Mar Biol 157:2109-2115

Dorrough J, Ash JE (1999) Using past and present habitat to predict the current distribution and abundance of a rare cryptic lizard, Delma impar (Pygopodidae). Austral Ecol 24:614-624 
Drummond AJ, Rambaut A (2007) BEAST: Bayesian evolutionary analysis by sampling trees. BMC Evol Biol 7:214

Ellstrand NC, Elam DR (1993) Population genetic consequences of small population size: implications for plant conservation. Annu Rev Ecol Syst 24:217-241

Evanno G, Regnaut S, Goudet J (2005) Detecting the number of clusters of individuals using the software STRUCTURE: a simulation study. Mol Ecol 14:2611-2620

Foll M, Gaggiotti OE (2006) Identifying the environmental factors that determine the genetic structure of populations. Genetics 174:875-891

Foll M, Gaggiotti O (2008) A genome-scan method to identify selected loci appropriate for both dominant and codominant markers: a Bayesian perspective. Genetics 180:977-993

Frankham R (1996) Relationship between genetic variation and populations size in wildlife. Conserv Biol 10:1500-1508

Frankham R (2005) Genetics and extinction. Biol Conserv 126:131-140

Frankham R (2015) Genetic rescue of small inbred populations: metaanalysis reveals large and consistent benefits of gene flow. Mol Ecol 24:2610-2618

Frankham R, Ballou JD, Eldridge MDB, Lacy RC, Ralls K, Dudash MR et al. (2011) Predicting the probability of outbreeding depression. Conserv Biol 25:465-475

Frankham R, Ballou JD, Ralls K, Eldridge MDB, Dudash MR, Fenster CB, et al. (2017) Genetic management of fragmented animal and plant populations, 1st edn. Oxford University Press, Oxford

Frankham R, Bradshaw CJA, Brook BW (2014) Genetics in conservation management: Revised recommendations for the 50/500 rules, Red List criteria and population viability analyses. Biol Conserv 170:56-63

Frankham R, Lees K, Montgomery ME, England PR, Lowe EH, Briscoe DA (1999) Do population size bottlenecks reduce evolutionary potential? Anim Conserv 2:255-260

Georges A, Gruber B, Pauly GB, White D, Adams M, Young MJ et al. (2018) Genomewide SNP markers breathe new life into phylogeography and species delimitation for the problematic shortnecked turtles (Chelidae: Emydura) of eastern Australia. Mol Ecol 27:5195-5213

Glémin S, Gaude T, Guillemin ML, Lourmas M, Olivieri I, Mignot A (2005) Balancing selection in the wild: testing population genetics theory of self-incompatibility in the rare species Brassica insularis. Genetics 171:279-289

Goudet J (2005) HIERFSTAT, a package for R to compute and test hierarchical F-statistics. Mol Ecol Resour 5:184-186

Gruber B, Unmack PJ, Berry OF, Georges A (2018) DARTR: an R package to facilitate analysis of SNP data generated from reduced representation genome sequencing. Mol Ecol Resour 18:691-699

Jaccoud D, Peng K, Feinstein D, Kilian A (2001) Diversity arrays: a solid state technology for sequence information dependent genotyping. Nucl Acids Res 29:e25

Janes JK, Malenfant M, Andrew RL, Miller JM, Dupuis JR, Gorrell JC et al. (2017) The $\mathrm{K}=2$ conundrum. Mol Ecol 26:3594-3602

Jones RN (1997) The biogeography of the grasses and lowland grasslands of south-eastern Australia. Adv Nat Conserv 2:11-18

Kamvar ZN, Brooks JC, Grünwald NJ (2015) Novel R tools for analysis of genome-wide population genetic data with emphasis on clonality. Front Genet 6:1-10

Knapp EE, Rice KJ (1996) Genetic structure and gene flow in Elymus glaucus (blue wildrye): implications for native grassland restoration. Restor Ecol 4:1-10

Kopelman NM, Mayzel J, Jakobsson M, Rosenberg NA, Ro AY (2015) CLUMPAK: a program for identifying clustering modes and packaging population structure inferences across K. Mol Ecol Resour 15:1179-1191

Kronenberger JA, Funk WC, Smith JW, Fitzpatrick SW, Angeloni LM, Broder ED et al. (2017) Testing the demographic effects of divergent immigrants on small populations of Trinidadian guppies. Anim Conserv 20:3-11

Lande R, Shannon S (1996) The role of genetic variation in adaptation and population persistence in a changing environment. Evolution (NY) 50:434-437

Liddell E, Cook CN, Sunnucks P (2020) Evaluating the use of risk assessment frameworks in the identification of population units for biodiversity conservation. Wildl Res 47:208-216

Lippé C, Dumont P, Bernatchez L (2006) High genetic diversity and no inbreeding in the endangered copper redhorse, Moxostoma hubbsi (Catostomidae, Pisces): the positive sides of a long generation time. Mol Ecol 15:1769-1780

Lloyd MW, Burnett RK, Engelhardt KAM, Neel MC (2011) The structure of population genetic diversity in Vallisneria Americana in the Chesapeake Bay: implications for restoration. Conserv Genet 12:1269-1285

Mable BK, Robertson AV, Dart S, Di Berardo C, Witham L (2005) Breakdown of self-incompatibility in the perennial Arabidopsis lyrata (Brassicaceae) and its genetic consequences. Evolution (NY) 59:1437-1448

Markgraf V, McGlone M, Hope G (1995) Neogene paleoenvironmental and paleoclimatic change in southern temperate ecosystems - a southern perspective. Trends Ecol Evol 10:143-147

Melville J, Goebel S, Starr C, Keogh JS, Austin JJ (2007) Conservation genetics and species status of an endangered Australian dragon, Tympanocryptis pinguicolla (Reptilia: Agamidae). Conserv Genet 8:185-195

Mijangos JL, Pacioni C, Spencer PBS, Craig MD (2015) Contribution of genetics to ecological restoration. Mol Ecol 22:22-37

Morgan JW (1995) Ecological studies of the endangered Rutidosis leptorrhynchoides: I. Seed production, soil seed bank dynamics, population density and their effects on recruitment. Aust J Bot 43:1-11

Moritz C (1999) Conservation units and translocations: Strategies for conserving evolutionary processes. Hereditas 130:217-228

Murray BG, Young AG (2001) Widespread chromosome variation in the endangered grassland forb Rutidosis leptorrhynchoides F. Muell. (Asteraceae: Gnaphalieae). Ann Bot 87:83-90

NSW Office of Environment and Heritage (2012) National Recovery Plan for Button Wrinklewort Rutidosis leptorrhynchoides. NSW Office of Environment and Heritage, Hurstville

Nybom H, Bartish I (2000) Effects of life history traits and sampling strategies on genetic diversity estimates obtained with RAPD markers in plants. Perspect Plant Ecol Evol Syst 3:93-114

Pacioni C, Hunt H, Allentoft ME, Vaughan TG, Wayne AF, Baynes A et al. (2015) Genetic diversity loss in a biodiversity hotspot: ancient DNA quantifies genetic decline and former connectivity in a critically endangered marsupial. Mol Ecol 24:5813-5828

Pavlova A, Selwood P, Harrisson KA, Murray N, Quin B, Menkhorst $P$ et al. (2014) Integrating phylogeography and morphometrics to assess conservation merits and inform conservation strategies for an endangered subspecies of a common bird species. Biol Conserv 174:136-146

Pickrell JK, Pritchard JK (2012) Inference of population splits and mixtures from genome-wide allele frequency data. PLoS Genet $8: 1-17$

Pickup M, Field DL, Rowell DM, Young AG (2012) Predicting local adaptation in fragmented plant populations: Implications for restoration genetics. Evol Appl 5:913-924

Pickup M, Field DL, Rowell DM, Young AG (2013) Source population characteristics affect heterosis following genetic rescue of fragmented plant populations. Proc R Soc B Biol Sci 280:20122058

Pickup M, Young AG (2008) Population size, self-incompatibility and genetic rescue in diploid and tetraploid races of Rutidosis leptorrhynchoides (Asteraceae). Heredity (Edinb) 100:268-274 
Pimm SL, Jenkins CN, Abell R, Brooks TM, Gittleman JL, Joppa LN et al. (2015) The biodiversity of species and their rates of extinction, distribution, and protection. Science 344:1246752

Potter S, Neaves LE, Lethbridge M, Eldridge MDB (2020) Understanding historical demographic processes to inform contemporary conservation of an arid zone specialist: the yellowfooted rock-wallaby. Genes (Basel) 11:1-24

Powell JM (1969) The squatting occupation of Victoria 1834-60. Aust Geogr Stud 7:9-27

Pritchard JK, Stephens M, Donnelly P (2000) Inference of population structure using multilocus genotype data. Genetics 155:945-959

Pritchard JK, Wen W (2003) Documentation for STRUCTURE Software: Version 2.

Raj A, Stephens M, Pritchard JK (2014) fastSTRUCTURE: variational inference of population structure in large SNP data sets. Genetics 197:573-589

Ralls K, Ballou JD, Dudash MR, Eldridge MDB, Fenster CB, Lacy $\mathrm{RC}$ et al. (2018) Call for a paradigm shift in the genetic management of fragmented populations. Conserv Lett 11:1-6

Rambaut A, Drummond AJ, Xie D, Baele G, Suchard M (2018) Posterior summarisation in Bayesian phylogenetics using Tracer 1.7. Syst Biol 67:901-904

Rodger YS, Greenbaum G, Silver M, Bar-david S, Winters G (2018) Detecting hierarchical levels of connectivity in a population of Acacia tortilis at the northern edge of the species' global distribution: combining classical population genetics and network analyses. PLoS ONE 13:1-16

Rojas D, Lima AP, Momigliano P, Ivo P, Dudaniec RY, Sauer TC et al. (2020) The evolution of polymorphism in the warning coloration of the Amazonian poison frog Adelphobates galactonotus. Heredity 124:439-456

Scarlett NH, Parsons RF (1990) Conservation biology of the southern Australian daisy Rutidosis leptorrhynchoides. In: Clark TW, Seebeck JH (eds) Management and conservation of small populations. Chicago Zoological Society, Chicago, p 195-205

Sinclair SJ (2010) National recovery plan for the large-fruit groundsel Senecio macrocarpus. Department of Sustainability and Environment, Melbourne

Sjogren P, Wyoni PI (1994) Conservation genetics and detection of rare alleles in finite populations. Conserv Biol 8:267-270

Spalink D, Mackay R, Sytsma KJ (2019) Phylogeography, population genetics and distribution modelling reveal vulnerability of
Scirpus longii (Cyperaceae) and the Atlantic Coastal Plain Flora to climate change. Mol Ecol 28:2046-2061

Team RC (2018) R: a language and environment for statistical computing

Wagenius S, Lonsdorf E, Neuhauser C (2007) Patch aging and the SAllee effect: breeding system effects on the demographic response of plants to habitat fragmentation. Am Nat 169:383-397

Weaver JC (1996) Beyond the fatal shore: pastoral squatting and the occupation of Australia. Am Hist Rev 101:981-1007

Weeks AR, Sgro CM, Young AG, Frankham R, Mitchell NJ, Miller KA et al. (2011) Assessing the benefits and risks of translocations in changing environments: A genetic perspective. Evol Appl 4:709-725

Weeks AR, Stoklosa J, Hoffmann AA (2016) Conservation of genetic uniqueness of populations may increase extinction likelihood of endangered species: the case of Australian mammals. Front Zool 13:1-9

Weir BS, Cockerham CC (1984) Estimating F-statistics for the analysis of population structure. Evolution (NY) 38:1358-1370

Wells GP, Young AG (2002) Effects of seed dispersal on spatial genetic structure in populations of Rutidosis leptorrhychoides with different levels of correlated paternity. Genet Res 79:219-226

Whiteley AR, Fitzpatrick SW, Funk WC, Tallmon DA (2015) Genetic rescue to the rescue. Trends Ecol Evol 30:42-49

Young AG, Brown AHD, Murray BG, Thrall PH, Miller CH (2000) Genetic erosion, restricted mating and reduced viability in fragmented populations of the endangered grassland herb Rutidosis leptorrhynchoides. In: Young AG, Clarke G (eds) Genetics, demography and viability of fragmented populations, Cambridge University Press, London, p 335-359

Young AG, Brown AHD, Zich FC (1999) Genetic structure of fragmented populations of the endangered Daisy Rutidosis leptorrhynchoides. Cons Biol 13:256-265

Young AG, Miller C, Gregory E, Langston A (2000) Sporophytic selfincompatibility in diploid and tetraploid races of Rutidosis leptorrhynchoides (Asteraceae). Aust J Bot 48:667-672

Young AG, Murray BG (2000) Genetic bottlenecks and dysgenic gene flow into re-established populations of the grassland daisy, Rutidosis leptorrhynchoides. Aust J Bot 48:409-416

Young AG, Pickup M (2010) Low S-allele numbers limit mate availability, reduce seed set and skew fitness in small populations of a self-incompatible plant. J Appl Ecol 47:541-548 